\title{
A State Variable Approach for the Calculation of Switching Transients on a Power Transmission Line
}

\author{
ADAM SEMLYEN, SENIOR MEMBER, IEEE, AND MANSOUR H. ABDEL-RAHMAN, MEMBER, IEEE
}

\begin{abstract}
This paper gives the outline of a new method for the calculation of electromagnetic transients on transmission lines above homogeneous ground. The basic idea is that line transfer functions which normally are calculated in terms of hyperbolic functions of $\omega$ are now expressed in terms of $s=\sigma+j \omega$. This is possible due to the existence of analytical expressions of line impedances and admittances in terms of $j \omega$, or $s$ in the new approach. It is then possible to find the poles of the transfer function by the secant method and then the corresponding residues. The partial fraction approximation of the transfer functions is incomplete and a correction may be used to account for the missing poles. The calculations are performed numerically only for samples of $s$ needed for the calculation of poles and residues. The end result is a set of state equations with $v$ and $i$ as inputs/outputs at the switch terminals. Inputs and outputs are interchanged as the switch poles open or close. The integration can be numcrical or closed form for sinusoidal inputs (generator voltages) and the state variables carry the past information from one stage of switching to the next. Essential parts of the procedures are illustrated by numerical results.
\end{abstract}

\section{INTRODUCTION}

$\mathrm{T}$ HE SIMULATION of electromagnetic transients in a power system nctwork is of great importance for the design of line and station insulation and selection of equipment. Earlier programs considered the transmission lines lossless and traveling wave methods have been applied [1]. Frequency domain methods [2] have been used in conjunction with the Fourier transform to take into account the frequency dependence of transmission line parameters as quantified originally by Carson [3]. Later an Electro-Magnetic Transients Program (EMTP) has been developed, based on the procedure of [1], and has included frequency dependent modeling of transmission line parameters [4][7], while retaining the traveling wave approach as its basic procedure. The main advantage in using traveling waves is that geographically distant nodes are computationally separated at any given instant, due to the travel delay. The computations are at present performed sequentially but the problem is of reduced size and amenable to parallel processing. The disadvantages consist in the difficulty of creating reduced order system equivalents and in the requirement for small integration steps imposed by the discretization needed for traveling wave front representation. A step size of, say, $10^{-5} \mathrm{~s}$ leads to lengthy computations,

Manuscript received August 19, 1981; revised March 26, 1982. This paper was supported in part by the Natural Sciences and Engineering Research Council of Canada under a Grant, and in part by Ontario Hydro under a Research Contract.

The authors are with the Department of Electrical Engineering, University of Toronto, Toronto, Ontario, Canada M5S 1A4. with possible accumulation of roundoff errors, if the total study time is of, say, $1 \mathrm{~s}$. The transient may have lasted only about $10^{-2} \mathrm{~s}$. Because of this, and for theoretical reasons, there has been some evidence of interest in lumped parameter, rather than traveling wave modeling [8]. Such methods present, however, difficulties because of the need to represent by rational polynomials the transfer functions of transmission lines. Indeed, Carson's corrections [3] require numerical evaluations, and the transfer functions contain square roots and hyperbolic functions of logarithmic expressions and of Carson's corrections, so that a fitting procedure is very difficult.

The basic new idea of this paper consists in using the complex variable $s=\sigma+j \omega$, instead of $j \omega$, for frequency domain modeling. This has become possible due to very good closed form expressions for the line impedance $Z$ in terms of the complex penetration depth

$$
p=\frac{1}{\sqrt{j \omega \mu_{0} \kappa}}
$$

where $\kappa$ is the conductivity of earth. We simply replace $j \omega$ in (1) by $s$ and obtain $Z(s)$. Similarly, we write $Y(s)=G+$ $s C$ for the line admittance. Then all required transfer functions become functions of $s$.

The complex depth $p$ is used for resistive homogeneous earth return for establishing the position of an image return conductor. This is placed at a (complex) depth deeper by $2 p$ than its position with perfectly conducting earth [9]-[11]. The approximation, with Carson's equations as reference, has errors of at most a few percent [12] for all frequencies and conductor positions.

Once transfer functions are expressed in terms of $s$, thcy or their combinations can easily be approximated by rational polynomials and realized in the form of state equations with not too many state variables. Their integration can be done using variable step size and, when no nonlinearities are considered in the system, the integration can be performed even in closed form since the inputs are sinusoidal generator voltages.

These ideas and procedures constitute the contributions of this paper to the realization theory of overhead transmission lines. The paper gives the outline of the new method and illustrates its essential features. Many prob- 


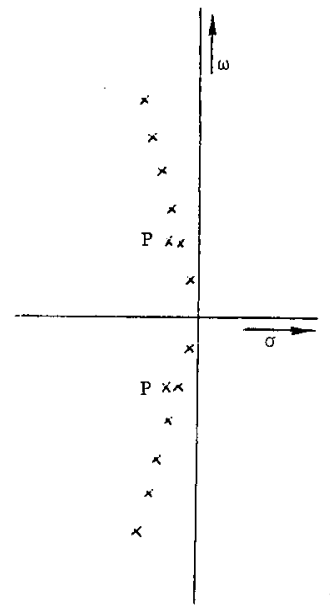

(a)

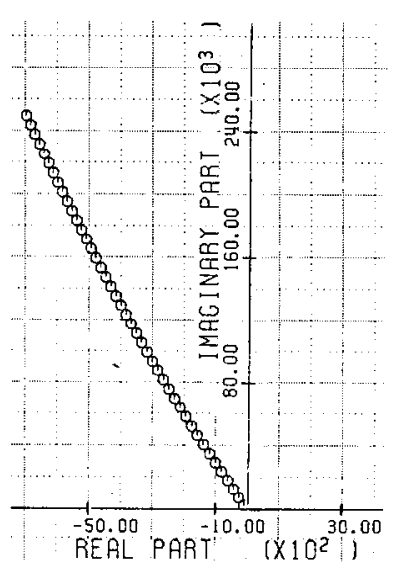

(b)

Fig. 1. Location of poles. (a) General case; $P$ represents hidden poles. (b) Poles of $H(s)=V_{2}(s) / E(s)$ of the system of Fig. 4; typically $\sigma \cong$ $0.03 \omega$.

lems of detail arc of routine numerical nature and successful procedures will only be indicated without explaining any details of implementation.

\section{OUTLINE OF METHOD}

In order to minimize analytical calculations, a transfer function $H\left(s_{k}\right)$ has to be calculated for a specific, numerically defined, complex frequency $s_{k}=\sigma_{k}+j \omega_{k}$. For this the following calculations are performed for a given $s_{k}$.

a) For all lines $Z$ and $Y$ are calculated; then a modal analysis is performed if the line is not single phase; and, finally, line transfer function matrices (TFM) are obtained.

b) The line transfer functions are assembled in a matrix for the whole system. This could be an admittance matrix for structured calculations in power system networks [8]. The matrix is reduced numerically eliminating nodes of no interest and retaining terminals for inputs, output, and nonlinear attached branches [8]. For simplicity we consider as end result a TFM, $H\left(s_{k}\right)$.

Thus $H\left(s_{k}\right)$ is the result of numerical evaluations. In

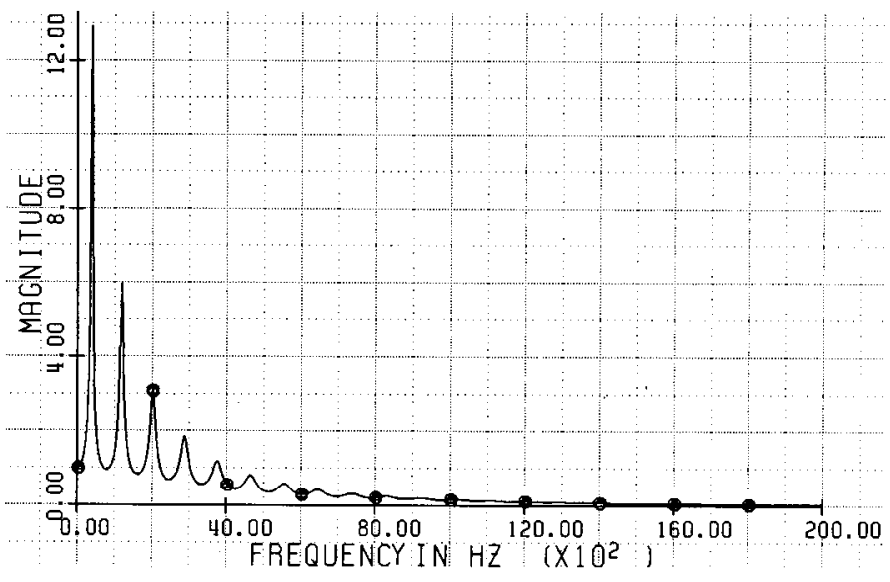

(a)

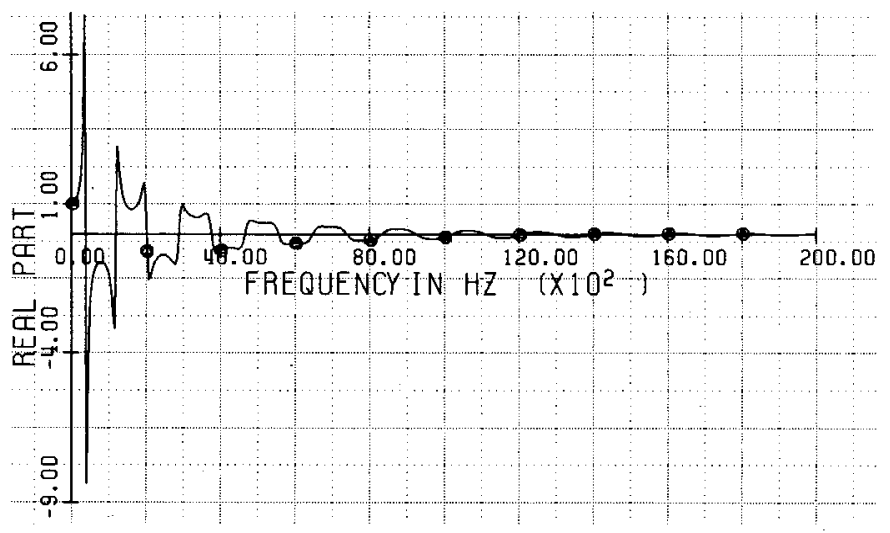

(b)

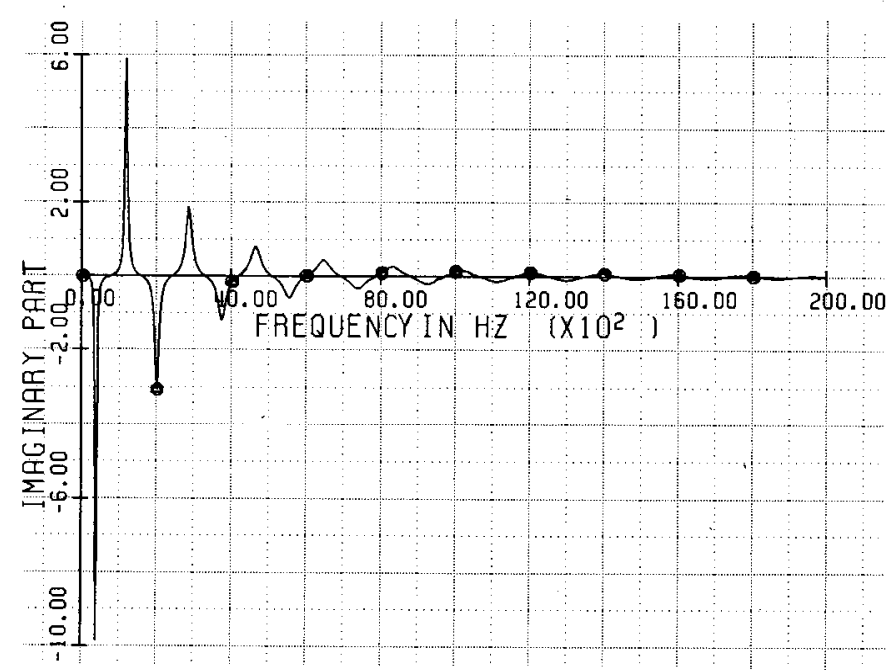

(c)

Fig. 2. $H(s)$ and $H_{R}(s)$ evaluated for $s=j \omega$ related to (13) and the circuit of Fig. 4. (a) Magnitude. (b) Real part. (c) Imaginary part. $\left.\begin{array}{cc}O & H(s) \\ \Delta & H_{R}(s)\end{array}\right\}$ practically superimposed.

simple cases, as the one used for illustration in this paper, $H(s)$ can be obtained in analytical form.

It has been found that the poles of $H(s)$ are close to the $\omega$-axis (Fig. 1). Fig. 2 shows profiles of $H(s)$ taken along the $\omega$-axis and Fig. 3 shows profiles along an axis $s=$ 


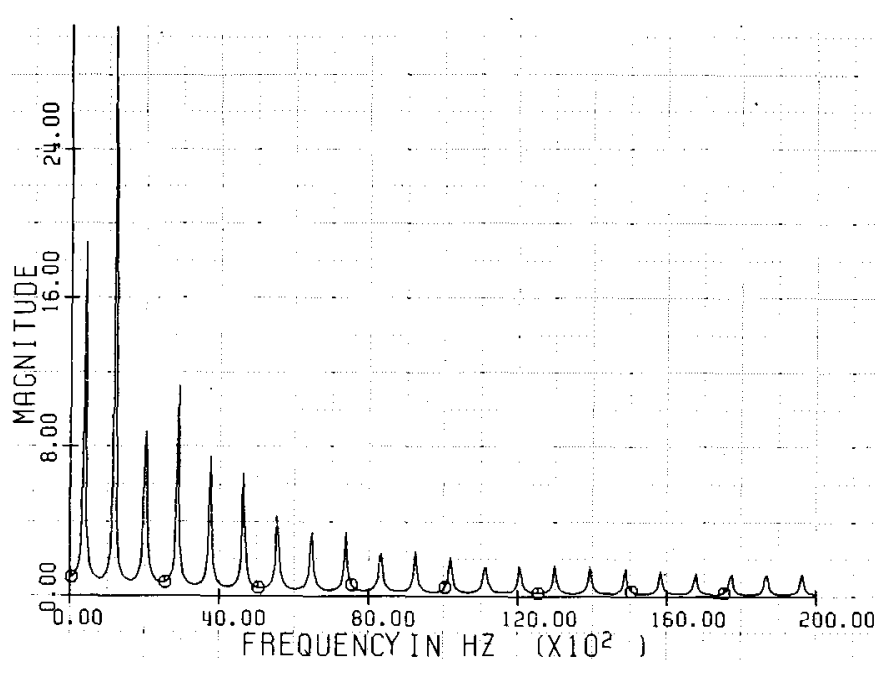

(a)

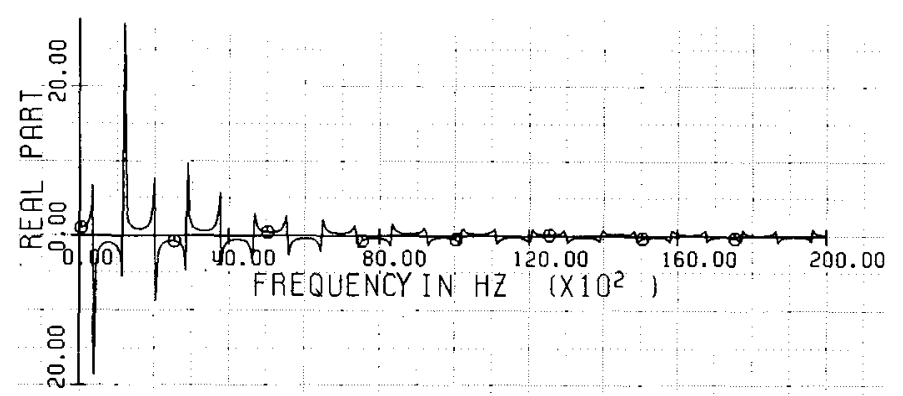

(b)

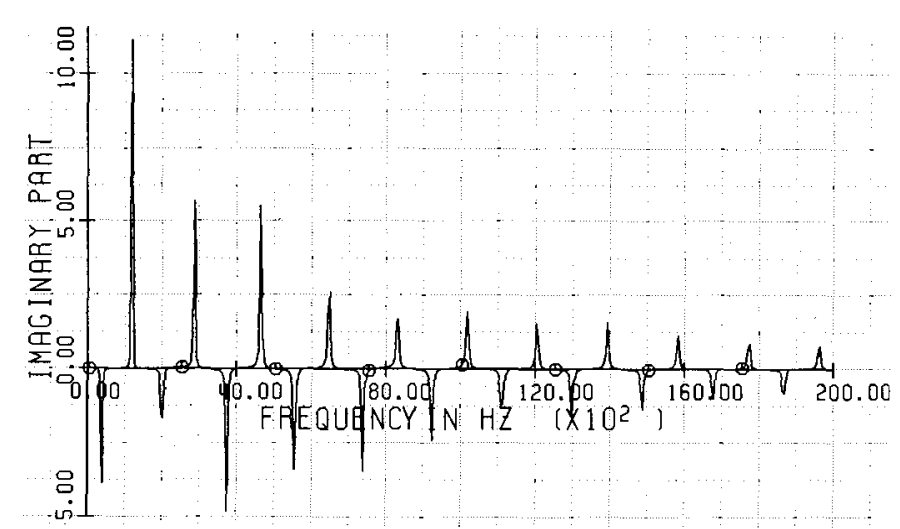

(c)

Fig. 3. Profile of $H(s)$ along $s=(-0.03+j) \omega$. (a) Magnitude. (b) Real part. (c) Imaginary part.

$(-0.03+j) \omega$ crossing the $s$-plane more closely to the peaks.

In order to locate the poles, we have developed the following procedure. The $\omega$-axis is first scanned (by computer) and the peaks of the profiles are located. On occasion poles are "hidden" from the $\omega$-axis (see poles $P$ in Fig. 1a): then the profiles of Fig. 3 are more useful. Two values of $s$ are taken, one before and one after the peak, $s_{1}$ and $s_{2}$, and $H\left(s_{1}\right)$ and $H\left(s_{2}\right)$ are evaluated. Then the secant method is used to iteratively determine the exact location of the pole. The desired number of poles is thus located.

The residues are calculated by a single evaluation of $H(s)$ very close to the pole.

For the poles at high frequencies, which are not calcu- lated, a single equivalent pole is evaluated. This yields a correction term for the missing poles.

The end result is a partial fraction approximation for the scalar (or TFM) $H(s)$. It is converted in a straightforward way into state equations

$$
\begin{aligned}
& \dot{x}=A x+B u \\
& y=C x+D u .
\end{aligned}
$$

If care is taken to have $|D| \neq 0$, inputs and outputs in (2), i.e., voltages and current can be interchanged, to follow the switching operations in appropriate sequence. The state variables remain the same. The integration of (2) is performed numerically or in closed form if $u$ is a sine function (vector).

\section{Calculation of Poles and Residues of Transfer FunCtions}

\section{A. Approximation to Carson's Formulas}

Consider the complex depth of a mirroring surface

$$
p=\frac{1}{\sqrt{s \mu_{0} \kappa}} .
$$

Then the mutual impedance between two conductors $i$ and $j$ at height $h$ and $h^{\prime}$, respectively, and at horizontal distance $D$ is [11], [12]

$$
Z_{i j}(s)=j \omega \frac{\mu_{0}}{2 \pi} \ln \frac{\sqrt{\left(h+h^{\prime}+2 p\right)^{2}+D^{2}}}{\sqrt{\left(h-h^{\prime}\right)^{2}+D^{2}}} .
$$

As shown in reference [12], expression (3) is remarkably accurate for most practical purposes.

If $i=j$ (3) reduces to the self impedance if the conductor impedance is added. This is also function of $s$ (see discussion to reference [7]).

Thus the line impedance $Z$ is function of $s$. It is a matrix in the case of a three-phase line. The same applies for $Y$, except that there $p=0$.

\section{B. Transfer Functions of Single Phase Lines for $s=s_{k}$}

Consider a line of length $l$ with voltages $V_{1}, V_{2}$ and injected currents $I_{1}, I_{2}$ at the terminals. The following TFM relations are pertinent:

$$
\begin{aligned}
{\left[\begin{array}{l}
I_{1} \\
I_{2}
\end{array}\right] } & =\left[\begin{array}{cc}
Y^{\prime} & Y^{\prime \prime} \\
Y^{\prime \prime} & Y^{\prime}
\end{array}\right]\left[\begin{array}{l}
V_{1} \\
V_{2}
\end{array}\right] \\
{\left[\begin{array}{l}
V_{1} \\
V_{2}
\end{array}\right] } & =\left[\begin{array}{ll}
Z^{\prime} & Z^{\prime \prime} \\
Z^{\prime \prime} & Z^{\prime}
\end{array}\right]\left[\begin{array}{l}
I_{1} \\
I_{2}
\end{array}\right] \\
{\left[\begin{array}{c}
V_{1} \\
I_{1}
\end{array}\right] } & =\left[\begin{array}{ll}
A & B \\
C & A
\end{array}\right]\left[\begin{array}{c}
V_{2} \\
-I_{2}
\end{array}\right] \quad\left(A^{2}-B C=1\right) \\
{\left[\begin{array}{c}
V_{1} \\
-I_{2}
\end{array}\right] } & =\left[\begin{array}{ll}
L & M \\
N & L
\end{array}\right]\left[\begin{array}{c}
V_{2} \\
I_{1}
\end{array}\right] \quad\left(L^{2}-M N=1\right) .
\end{aligned}
$$

Denote

$$
\gamma=\sqrt{Z(s) Y(s)}
$$


and

$$
Y_{c}=\sqrt{\frac{Y(s)}{Z(s)}} .
$$

Then

$$
\begin{aligned}
Y^{\prime} & =Y_{c} \frac{1}{\tanh \gamma l} \quad Y^{\prime \prime}=-Y_{c} \frac{1}{\sinh \gamma l} \\
Z^{\prime} & =Y_{c}^{-1} \frac{1}{\tanh \gamma l} \quad Z^{\prime \prime}=Y_{c}^{-1} \frac{1}{\sinh \gamma l} \\
A & =\cosh \gamma l \quad B=Y_{c}^{-1} \sinh \gamma l \quad C=Y_{c} \sinh \gamma l \\
L & =\frac{1}{\cosh \gamma l} \quad M=Y_{c}^{-1} \tanh \gamma l \quad N=-Y_{c} \tanh \gamma l .
\end{aligned}
$$

Clearly, the elements of all TFM's are functions of $\gamma$ and $Y_{c}$ of (5) and can be easily evaluated for any $s=s_{k}$. Only two such elements are independent.

We note at this stage that these functions are not rational in $s$. Hyperbolic functions in $x$ are rational, of infinite order, in $x$ and partial fraction expansion are given for such functions [14] with an infinite number but discrete poles. However, the hyperbolic functions in (6) are functions of $\gamma$, not of $s$. For all frequencies which are not close to zero, $\gamma$ is almost proportional to $s$ : therefore, for most frequencies of interest, the hyperbolic functions are close to rational. Also, for these frequencies, $Y_{c}$ is almost constant. Consequently, all functions (6) are close to rational functions in $s$, with a very large number of poles. This explains why the rational approximations used for these functions are so good.

\section{Transfer Functions of Three-Phase Lines for $s=s_{k}$}

For untransposed three-phase lines modal transformation matrices [13], [5] have to be calculated for any $s=s_{k}$. These are the eigenvector matrices $T_{V}$, and $T_{I}$ of $Z Y$ and $Y Z$, respectively, defined by

$$
\begin{gathered}
V=T_{V} V_{m} \\
I=T_{I} I_{m}
\end{gathered}
$$

where $V_{m}$ and $I_{m}$ are vectors of modal voltages and currents.

One can define modal impedance and admittance matrices by

$$
V_{m}=Z_{m} I_{m} \quad I_{m}=Y_{m} V_{m}
$$

or, using (7), by

$$
Z_{m}=T_{V}^{-1} Z T_{I} \quad Y_{m}=T_{I}^{-1} Y T_{V}
$$

The matrices $Z_{m}$ and $Y_{m}$ are diagonal and therefore the calculations in (5) and (6) can be performed directly (i.e., independently, for each mode). The result is $Y_{m}^{\prime}, Y_{m}^{\prime \prime}$, etc. Then the phase domain TFM results by using the definitions (7). For example, from (4a)

$$
I_{1}=Y^{\prime} V_{1} \text {. }
$$

Substitution of relations (7) into (9) yields

$$
Y^{\prime}=T_{1} Y_{m}^{\prime} T_{V}^{-1}
$$

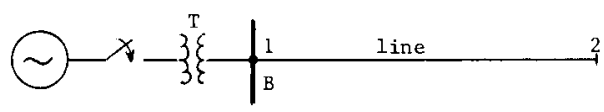

(a)

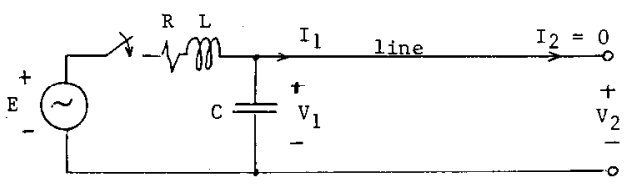

(b)

Fig. 4. Energization of open circuited single phase line. $T=$ transformer, $B=$ busbars with capacitance, $l=150 \mathrm{~km}, R=6.5 \Omega, L=0.034 \mathrm{H}$, $\mathrm{C}=0.005 \mu \mathrm{F}$. (a) General layout. (b) Circuit diagram.

Similarly

$$
Y^{\prime \prime}=T_{I} Y_{m}^{\prime \prime} T_{V}^{-1}
$$

In conclusion, for any $s=s_{k}$, we first calculate $T_{V}$ and $T_{I}$ as the eigenvector-matrices of $Z Y$ and $Y Z$, respectively. Then we calculate $Z_{m}$ and $Y_{m}$ using $\left(8^{\prime \prime}\right)$ and the desired modal TFM's using (6). Finally we convert these into the phase domain, as shown in (10).

\section{Transfer Functions of a Complex System for $s=s_{k}$}

The transfer function of a complex system can be obtained by assembling its components. For a larger system it is perhaps best to use a structured approach by means of a system nodal admittance matrix. Details of such a procedure are described, in another context, in reference [8]. Often, simpler cases are of interest and, then, no general procedure is needed. The example of the energization of the single phase line shown in Fig. 4 will be discussed next.

For the source network we can write, with the switch closed

$$
E=(1+(R+s L) s C) V_{1}+(R+s L) I_{1}
$$

and for the line equations (4c) and (6c) yield

$$
\begin{aligned}
& V_{1}=\cosh \gamma / V_{2} \\
& I_{1}=Y_{c} \sinh \gamma / V_{2} .
\end{aligned}
$$

Substitution of (12) into (11) gives the transfer function

$$
\begin{aligned}
H(s) & =\frac{V_{2}}{E} \\
& =\frac{1}{(1+(R+s L) s C) \cosh \gamma l+(R+s L) Y_{c} \sinh \gamma l} .
\end{aligned}
$$

This can easily be evaluated for any $s=s_{k}$.

\section{E. Calculation of Poles by the Secant method}

It is quite clear from the particular cases described that derivatives should not be calculated because of the complexity of the expressions. Therefore, the secant method [15] was used to obtain the poles of the transfer function $H(s)$. For this we define $F(s)=1 / H(s)$ and calculate the roots of $F(s)=0$. We found that the secant method worked well with complex variables (see also reference [16]). Its 
iteration algorithm is

$$
s_{k+2}=\frac{F\left(s_{k}\right) s_{k+1}-F\left(s_{k+1}\right) s_{k}}{F\left(s_{k}\right)-F\left(s_{k+1}\right)} .
$$

We have used double precision calculation because of the differences in (14) which may produce roundoff errors close to the root. It is interesting to note that poles in the $s$-plane could be reached with starting points on the $\omega$-axis. But, on occasions, a pole hidden behind other poles closer to the $\omega$-axis (see Fig. la) could not be reached even though it could be detected from the $\omega$-axis. As already mentioned, it was then found more reliable to do the searching and starting from a line slightly deviating from the pure imaginary $\omega$-axis (see Fig. 3).

It is also useful to emphasize here the significance of being able to evaluate $H(s)$ for a complex $s_{k}$. If this is not possible (as is the case in other approaches) and $H(s)$ can be evaluated only for $s=j \omega_{k}$, then we have the problem of rational approximation

$$
H(s)=C \frac{\pi\left(s-z_{i}\right)}{\pi\left(s-p_{j}\right)}
$$

with the zeros $z_{i}$ and poles $p_{j}$ to be determined so that (15) is satisfied for a given $H(s)$ at a sufficient number $N$ of imaginary values $s=j \omega_{k}$. This is equivalent to solving (perhaps in a least squares sense) $N$ nonlinear equations, in $z_{i}$ and $p_{j}$. The problem is difficult and often produces unstable solutions $\left(z_{i}, p_{i}\right.$ in the right half-plane) even if $H(s)$ is stable. With the secant method, using complex $s_{k}$, an arbitrary number of poles can be accurately located, usually with very little computer time (of the order of a few seconds).

\section{F. Calculation of Residues}

Consider the partial fraction expansion

$$
H(s)=\sum_{j=1}^{\infty} \frac{r_{j}}{s-p_{j}}
$$

equivalent to (15). The evaluation of the poles $p_{j}$ has been discussed in the previous section.

The residue $r_{j}$ can be obtained from

$$
r_{j}=\lim _{s \rightarrow p_{j}}\left\{H(s)\left(s-p_{j}\right)\right\} .
$$

The usual concern with (17) is that, at the limit, $r_{j}=\infty \times 0$ so that derivatives are normally used if the denominator of $H(s)$ is not available in factorized form. But since we want to avoid derivatives we evaluate (17) at some value $s$ very close to $p_{j}$. It does not matter from which direction we approach $p_{j}$ but for better accuracy double precision arithmetic is recommended.

The end result of these calculations is a rational approximation $H_{R}(s)$, in partial fraction expansion, of the original transfer function (or TFM) $H(s)$. Fig. 2 gives an example of $H(s)$ and its rational approximation $H_{R}(s)$, evaluated for $s=j \omega$, for the transfer function (13) related to the circuit of Fig. 4.

\section{The Rational Transfer Function}

\section{A. Effect of Truncation}

The rational transfer function

$$
H_{R}(s)=\sum_{j=1}^{n} \frac{r_{j}}{s-p_{j}}
$$

has $n$ poles identical to the first $n$ poles of the original function $H(s)$. Because of the missing poles and also because $H(s)$ is only close to being rational itself, $H_{R}(j \omega)$ only approximates $H(j \omega)$. The difference is so small that it can not be seen in Fig. 2 where both $H(j \omega)$ and $H_{R}(j \omega)$ are plotted.

One can note that $\left(16^{\prime}\right)$ is strictly proper even if the original function $H(s)$ is proper. The examples of possible transfer functions of equations (6) indicate that they can be of all types: strictly proper, such as $L=1 / \cos \gamma l$; proper, such as $Y^{\prime}=Y_{c} / \tanh \gamma l$; nonproper, such as $A=\cosh \gamma l$. Their nature results from their value at $s=\infty$.

If the original function is proper the approximation $\left(16^{\prime}\right)$ becomes more and more accurate for smaller values of $s=j \omega$ but, of course, at $s=j \infty H_{R} \rightarrow 0$ while $H \rightarrow H_{\infty} \neq 0$.

One will then seek a correction term for $\left(16^{\prime}\right)$ to account for the missing terms and to make $H_{R}(s)$ proper if that was the nature of the original function or to keep $H_{R}(s)$ strictly proper if that is the case. Nonproper transfer functions have not been considered.

The case of proper transfer functions is very important. If $H(s)$ is proper, then so is $1 / H(s)$, the inverse transfer function for which input and output are interchanged [17]. The input may be a voltage, the output a current when the switch pole is closed but, when the switch pole is open the two have to be interchanged. Input/output interchange is only possible if $H(s)$ is proper and, correspondingly, $|D| \neq 0$ in the state equation realization (2). Therefore, if $H(s)$ is proper, it is important to have the approximation $H_{R}(s)$ of (16') corrected to become also proper.

The aggregate value of the effect of missing poles $p_{i}=\sigma_{i}$ $+j \Omega_{i}\left(\sigma_{i}<0\right)$, evaluated at $s=j \omega$ for $\omega \ll \Omega_{i}$, is

$$
\Delta H(j \omega)=\sum_{i=n+1}^{\infty}\left(\frac{r_{i}^{\prime}+j r_{i}^{\prime \prime}}{j \omega-\left(\sigma_{i}+j \Omega_{i}\right)}+\frac{r_{i}^{\prime}-j r_{i}^{\prime \prime}}{j \omega-\left(\sigma_{i}-j \Omega_{i}\right)}\right) .
$$

The linear part of the Taylor series expansion of $\left(18^{\prime}\right)$ in terms of $j \omega$ is

$$
\Delta H(j \omega) \cong a+j b \omega
$$

where $a$ and $b$ are real constants.

Equation (18") shows that the error due to the missing terms is approximately constant in the plot of $\operatorname{Re}\{H(j \omega)\}$. This appears clearly in Fig. 5(b) where $H(s)=\tanh \gamma(s) l$ -1 . Equation (18") also shows that the error in the plot of $\operatorname{Im}\{H(j \omega)\}$ is approximately proportional to $\omega$, but this error is small and therefore not perceptible in Fig. 5(c).

The correction term for equation $\left(16^{\prime}\right)$ should be adequate if it provides a compensation as shown in equation $\left(18^{\prime \prime}\right)$. 


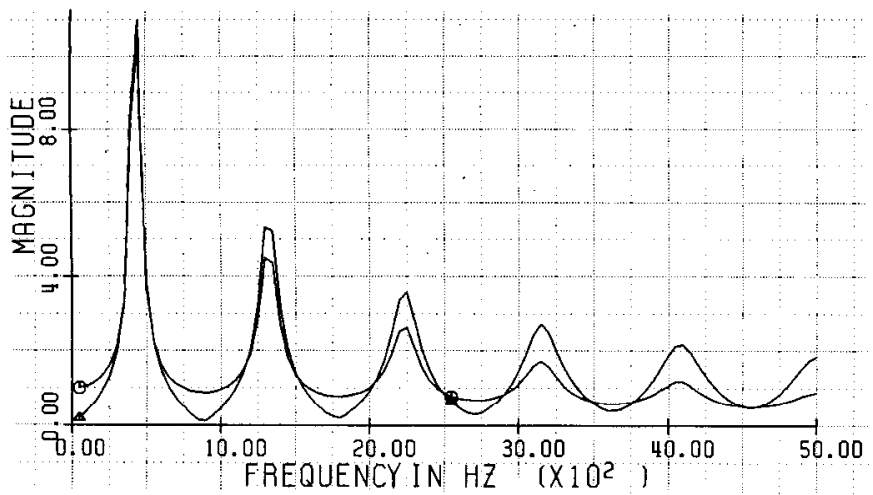

(a)

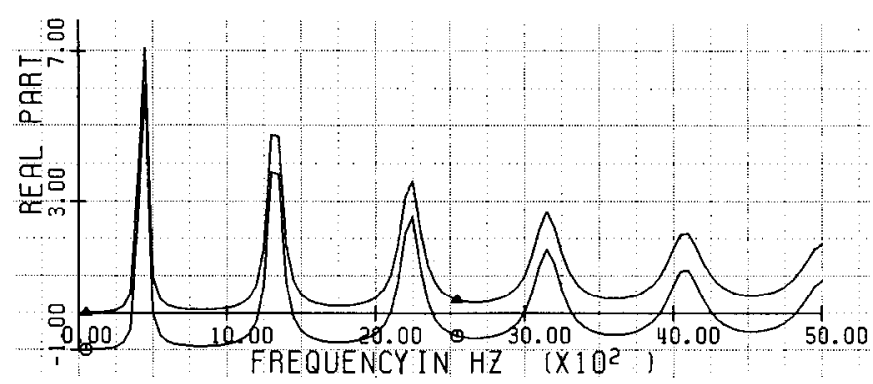

(b)

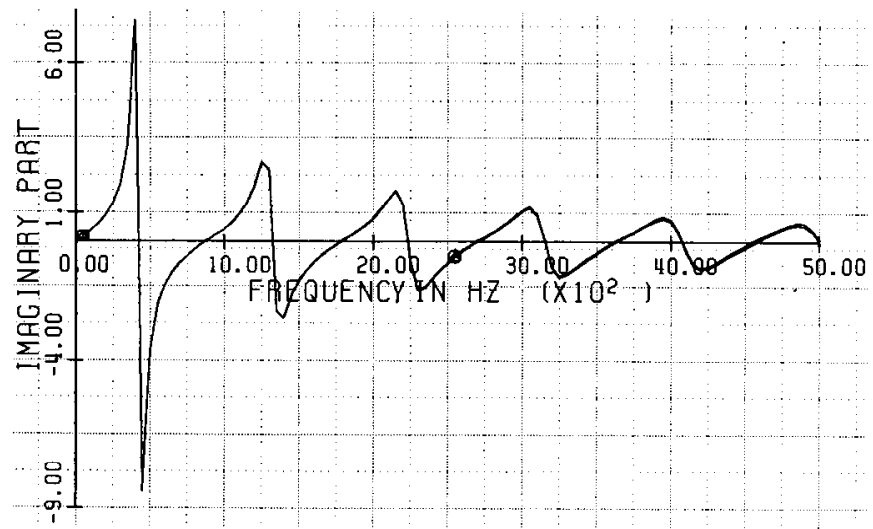

(c)

Fig. 5. Profile of $H(s)=\tanh \gamma(s) l-1$ along $s=j \omega$. (a) Magnitude. (b) Real part. (c) Imaginary part. $O H(j \omega) . \Delta H_{K}(j \omega)$.

It is not intended to calculate the constants $a$ and $b$ in $\left(18^{\prime \prime}\right)$ from information about the missing poles. Equation $\left(18^{\prime \prime}\right)$ simply indicates that a correction term should produce a close to linear variation of $\Delta H(j \omega)$. It should, therefore, have two parameters for this purpose, as has $\left(18^{\prime \prime}\right)$, with probably a pole at high frequency.

Fig. 6 gives the result of a correction term being added to the $H_{R}(j \omega)$ for the example of Fig. 5. This corresponds to a strictly proper transfer function and the details of the calculations are given in the next section.

\section{B. Correction for Missing Poles for Strictly Proper Transfer Functions}

We write the correction in the strictly proper form

$$
\Delta H(s)=\frac{r_{\infty}}{s-p_{\infty}}
$$

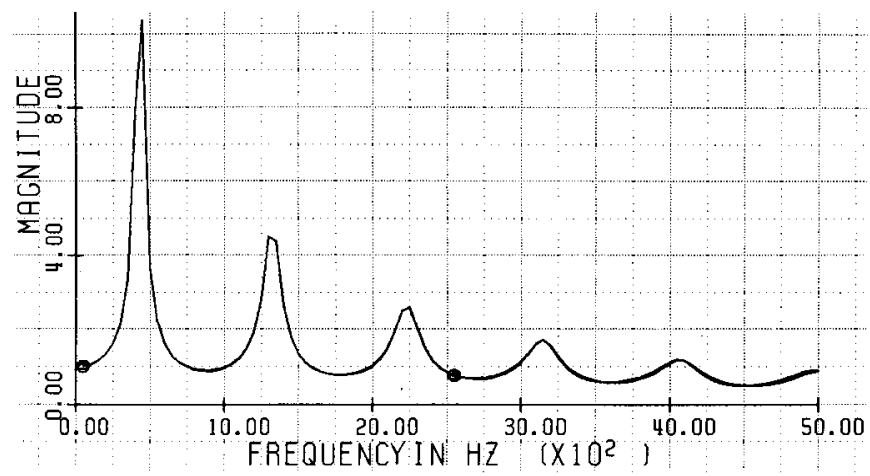

(a)

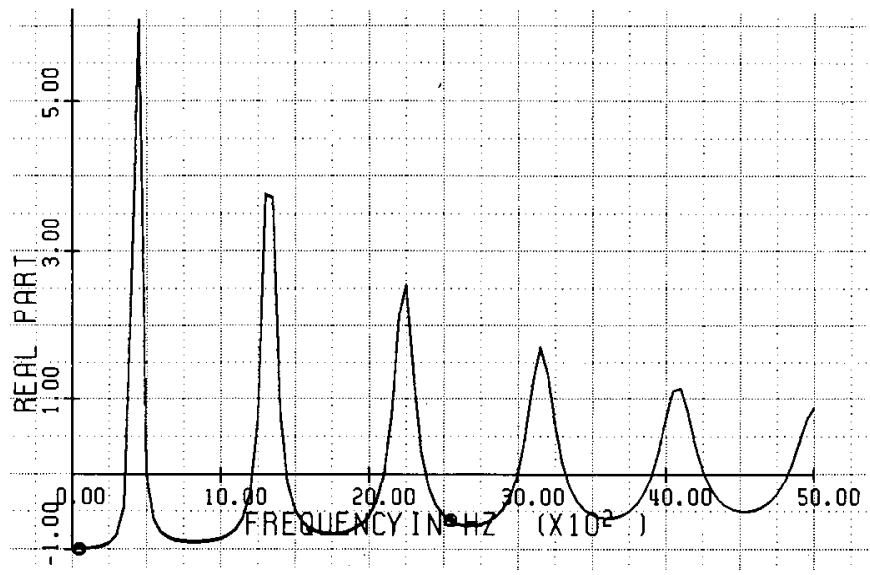

(b)

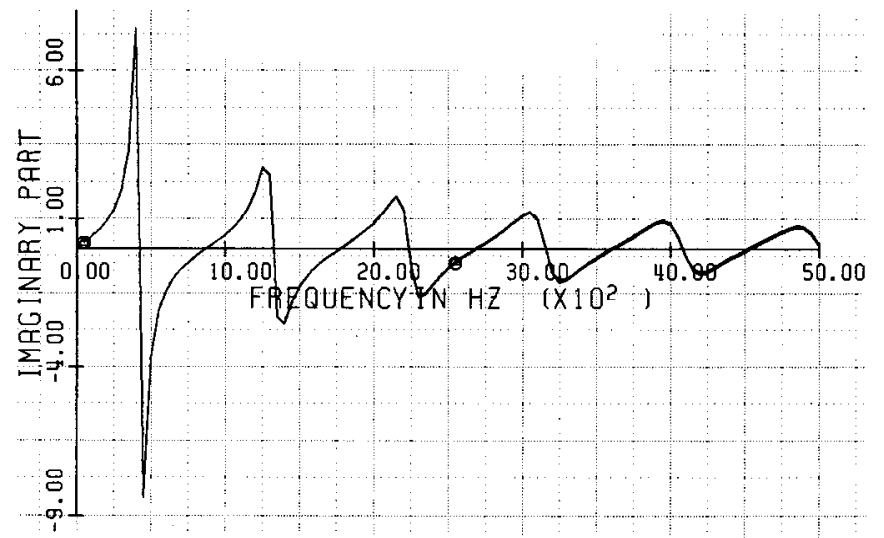

(c)

Fig. 6. Profile of $H(s)=\tanh \gamma(s) l-1$ along $s=j \omega$ (with correction).

(a) Magnitude. (b) Real part. (c) Imaginary part. $\left.\begin{array}{cc}\bigcirc & H(j \omega) \\ \Delta & H_{R}(j \omega)\end{array}\right\}$ practically superimposed.

with $r_{\infty}$ and $p_{\infty}$ assumed to be real.

From (19) and (18") we obtain for $s=0$

$$
-\frac{r_{\infty}}{p_{\infty}}=\Delta H(0)
$$

and for $s=j \omega_{\max }$, where $\omega_{\max }$ is the highest frequency of interest, we obtain

$$
\frac{r_{\infty}}{j \omega_{\max }-p_{\infty}}=\Delta H\left(j \omega_{\max }\right) .
$$




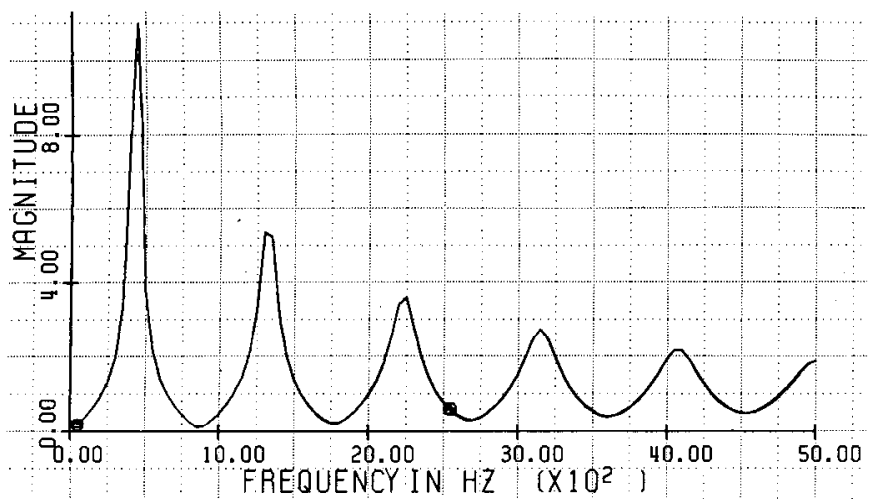

(a)

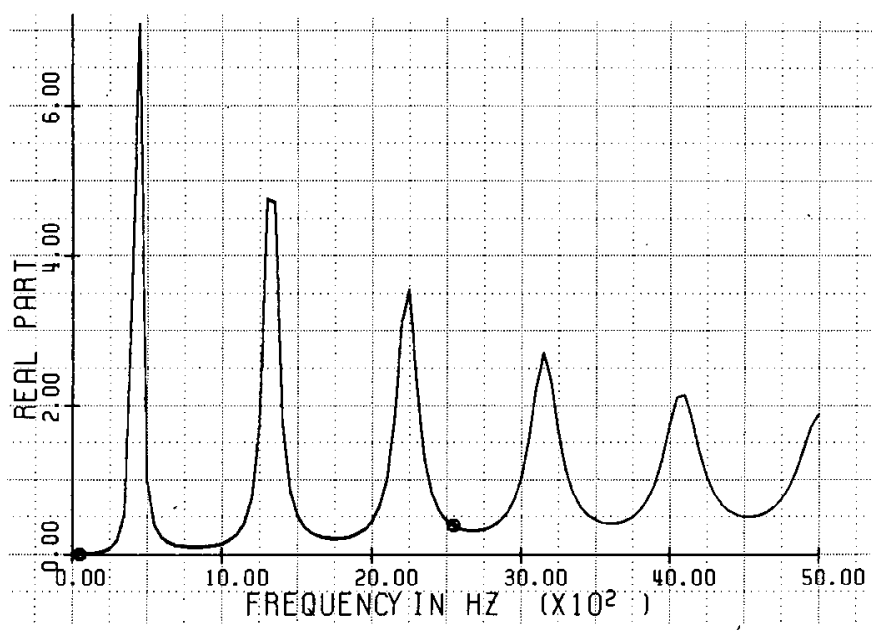

(b)

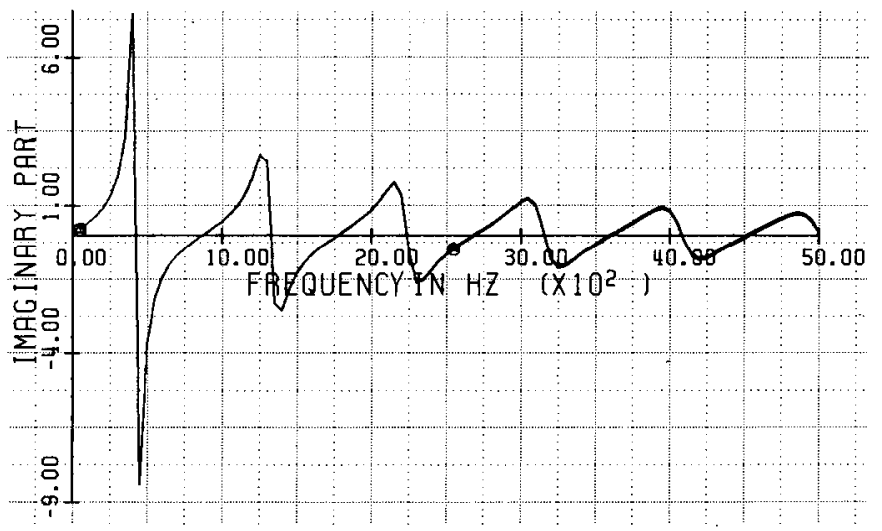

(c)

Fig. 7. Profile of $H(s)=\tanh \gamma(s) l$ along $s=j \omega$. (a) Magnitude. (b) Real part. (c) Imaginary part. $\left.\begin{array}{cc}0 & H(j \omega) \\ \Delta & H_{R}(j \omega)\end{array}\right\}$ practically superimposed.

This yields, for $p_{\infty} \gg \omega_{\max }$ and using equation $\left(20^{\prime}\right)$

$$
p_{\infty}=\omega_{\max } \frac{\Delta H(0)}{\operatorname{Im}\left\{\Delta H\left(j \omega_{\max }\right)\right\}}
$$

and

$$
r_{\infty}=-p_{\infty} \Delta H(0) .
$$

Thus the correction (19) is calculated by using only two values of the approximation error $\Delta H(s)=H(s)-H_{R}(s)$,
$\Delta H(0)$ and $\Delta H\left(j \omega_{\max }\right)$ evaluated at $s=0$ and $s=j \omega_{\max }$, respectively.

\section{Correction for Missing Poles for Proper Transfer} Functions

We write the correction in the form

$$
\Delta H(s)=H_{\infty} \frac{s-z_{\infty}}{s-p_{\infty}}
$$

so that the corrected transfer function $H_{R}(s)$ of $\left(16^{\prime}\right)$ becomes proper, with the value $H_{\infty}$ for $s=j \infty$.

For $s=0$ (22) gives

$$
\frac{z_{\infty}}{p_{\infty}}=\frac{\Delta H(0)}{H_{\infty}}
$$

and for $s=j \omega_{\max }(22)$ becomes

$$
H_{\infty} \frac{j \omega_{\max }-z_{\infty}}{j \omega_{\max }-p_{\infty}}=\Delta H\left(j \omega_{\max }\right) .
$$

The solution of (23) is

$$
\begin{aligned}
p_{\infty} & =\operatorname{Re}\left\{j \omega_{\max } \frac{\Delta H\left(j \omega_{\max }\right)-H_{\infty}}{\Delta H\left(j \omega_{\max }\right)-\Delta H(0)}\right\} \\
& \cong \omega_{\max } \frac{\Delta H(0)-H_{\infty}}{\operatorname{Im}\left\{\Delta H\left(j \omega_{\max }\right)\right\}} \\
z_{\infty} & =p_{\infty} \frac{\Delta H(0)}{H_{\infty}} .
\end{aligned}
$$

Fig. 7 shows the fitting for the proper transfer function $H(s)=\tanh \gamma(s) l . H_{R}(s)$ is not corrected and a correction does not appear to be necessary in this particular case.

\section{State Equations and Thfir Integration}

\section{A. Interchanging Input and Output Variables}

Assume that in (2) $u$ is the vector of voltages and $y$ the vector of currents. As an example consider the energization of a three-phase line (Fig. 8) by sequentially closing the poles of a breaker. When pole $a$ is closed the inputs are $u_{a}, y_{b}=0, y_{c}=0$; when poles $a$ and $b$ are closed the inputs are $u_{a}, u_{b}, y_{c}=0$; and when finally all three poles are closed the inputs are $u_{a}, u_{b}, u_{c}$. When, subsequently, all three poles are open the inputs are $y_{a}=0, y_{b}=0, y_{c}=0$. This example shows that some or all of the inputs and outputs have to be interchanged.

The mathematical procedure is shown for a complete interchange (inverse system [17]) in the state equations (2). If $D$ is nonsingular equation ( $\left.2^{\prime \prime}\right)$ yields

$$
u=-D^{-1} C x+D^{-1} y .
$$

Substitution of $\left(25^{\prime}\right)$ with $\left(2^{\prime}\right)$ yields

$$
\dot{x}=\left(A-B D^{-1} C\right) x+B D^{-1} y .
$$

Equations (25) can be rewritten as

$$
\begin{aligned}
& \dot{x}=A^{\prime} x+B^{\prime} y \\
& u=C^{\prime} x+D^{\prime} y
\end{aligned}
$$




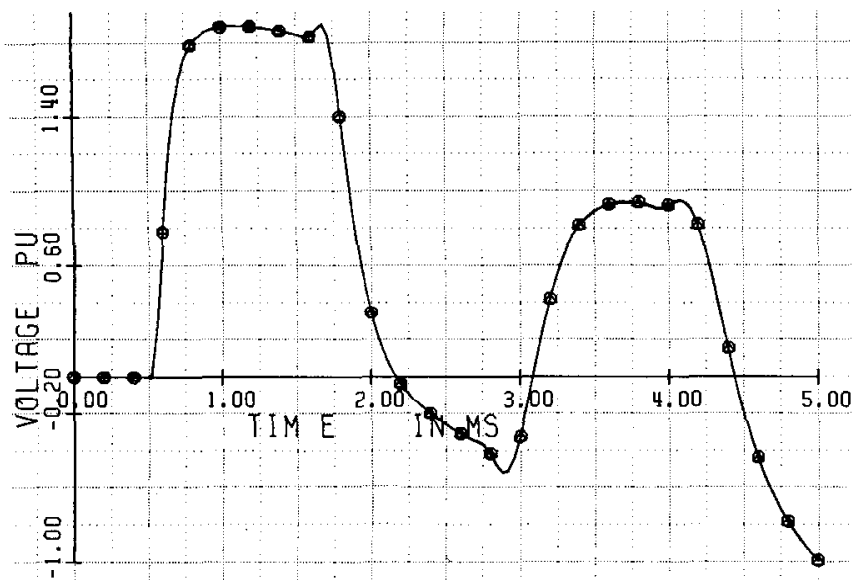

Fig. 8. Receiving end voltage transient for the system of Fig. 4. $\begin{array}{rr}\Delta & \text { Inverse Fourier transform solution } \\ \Delta & \text { Closed form solution }\end{array}$ practically superimposed.

where

$$
A^{\prime}=A-B D^{-1} C \quad B^{\prime}=B D^{-1} \quad C^{\prime}=-D^{-1} C \quad D^{\prime}=D^{-1} .
$$

It can be seen from (26) that the inversion does not affect the state variables, but all coefficient matrices are changed; the natural modes of the inverse system are changed too because $A$ and $A^{\prime}$ have different eigenvalues.

\section{B. Initialization for Sinusoidal Variables}

A transient will often start from a sinusoidal steady-state with frequency $\omega_{0}$. It is then easy to obtain the initial value $x(0)$ of the state variables $x$ of equation $\left(2^{\prime}\right)$. For this purpose we represent the input voltages

$$
u_{i}=U_{i_{\max }} \sin \left(\omega_{0} t+\phi_{i}\right)
$$

by phasors

$$
\bar{U}_{i}=U_{i_{\max }} e^{j \phi_{i}} .
$$

In equation $\left(2^{\prime}\right)$ we represent $x$ also by phasors. Equation (2') yields

$$
\bar{X}=\bar{G} \bar{U}
$$

where

$$
\bar{G}=\left(j \omega_{0} I-A\right)^{-1} B .
$$

One element of $\left(29^{\prime}\right)$ is

$$
\bar{X}_{k}=\sum_{i} \bar{G}_{k i} \bar{U}_{i}
$$

with

$$
\bar{G}_{k i}=G_{k i} e^{j \theta_{k i}} .
$$

In the time domain $\left(30^{\prime}\right)$ gives

$$
x_{k}(t)=\sum_{i} G_{k i} U_{i_{\max }} \sin \left(\omega_{0} t+\phi_{i}+\theta_{k i}\right) .
$$

Equation (31) gives the initial value of $x_{k}$ for $t=0$.

\section{Closed-Form Solution for Sinusoidal Input}

We consider first the single input case for equation $\left(2^{\prime}\right)$

$$
\dot{x}=A x+b u
$$

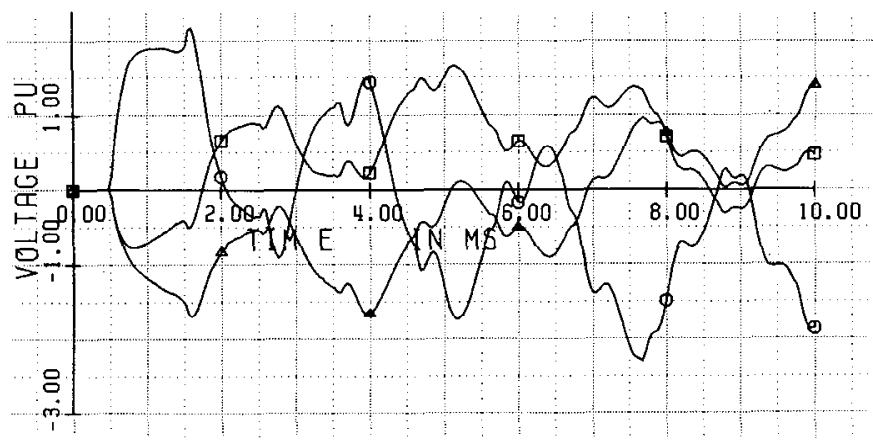

Fig. 9. Receiving end voltage transient at simultaneous energization of a three phase line, $150 \mathrm{~km}, 500 \mathrm{kV}$, transposed, source inductance $=0.034$ H. O Phase $a$. $\Delta$ Phase $b$. $\square$ Phase $c$

where

$$
u=U_{\max } \sin \left(\omega_{0} t+\phi\right) .
$$

In equation $\left(32^{\prime}\right)$ the vector $x(0)$ is known from the value of $x(t)$ at the end of the previous stage of the transient or from the sinusoidal steady-state, discussed in the previous section.

We note that $A$ is diagonal since (32') has been obtained from $H_{R}(s)$ in the form of a series of simple fractions. It has the poles $p_{j}$ on its diagonal and the vector $b$ consists of the residues $r_{j}$ of the simple fractions.

Integration of (32) yields for state variable $x_{j}$ related to a single partial fraction

$$
\begin{aligned}
x_{j}(t)= & e^{p_{j} t} x_{j}(0) \\
& -U_{\max } \frac{r_{j}}{\omega_{0}^{2}+p_{j}^{2}}\left[p_{j} \sin \left(\omega_{0} t+\phi\right)+\omega_{0} \cos \left(\omega_{0} t+\phi\right)\right. \\
& \left.-e^{p_{j} t}\left(p_{j} \sin \phi+\omega_{0} \cos \phi\right)\right] .
\end{aligned}
$$

We note that, if $p_{j}$ and $r_{j}$ are complex, they appear in conjugate pairs and so will $x_{j}(t)$. Therefore the resultant output will be real, as expected.

Fig. 8 represents the receiving end voltage transient resulting from the frequency domain data of Fig. 2 corresponding to the energization of the line shown in Fig. 4. One plot corresponds to the closed form integration of the state equations derived from $H_{R}(s)$. The other plot represents the inverse Fourier transform of the original $H(j \omega)$. As can be seen, the agreement is very good.

Fig. 9 gives an example for the closed form calculation of transients on a three-phase transmission line. Because of the simultaneous pole closing, chosen for simplicity, there is no ground mode damping in this case. Fig. 10 illustrates the case of sequential energization.

The total computer CPU time, including the rational fitting, is of the order of a few seconds. The storage requirements are very low.

\section{Numerical Integration for Systems with Nonlinear Elements}

For nonlinear elements connected to the network we will consider special terminals with inputs $u^{\prime}$ and outputs $y^{\prime}$, 

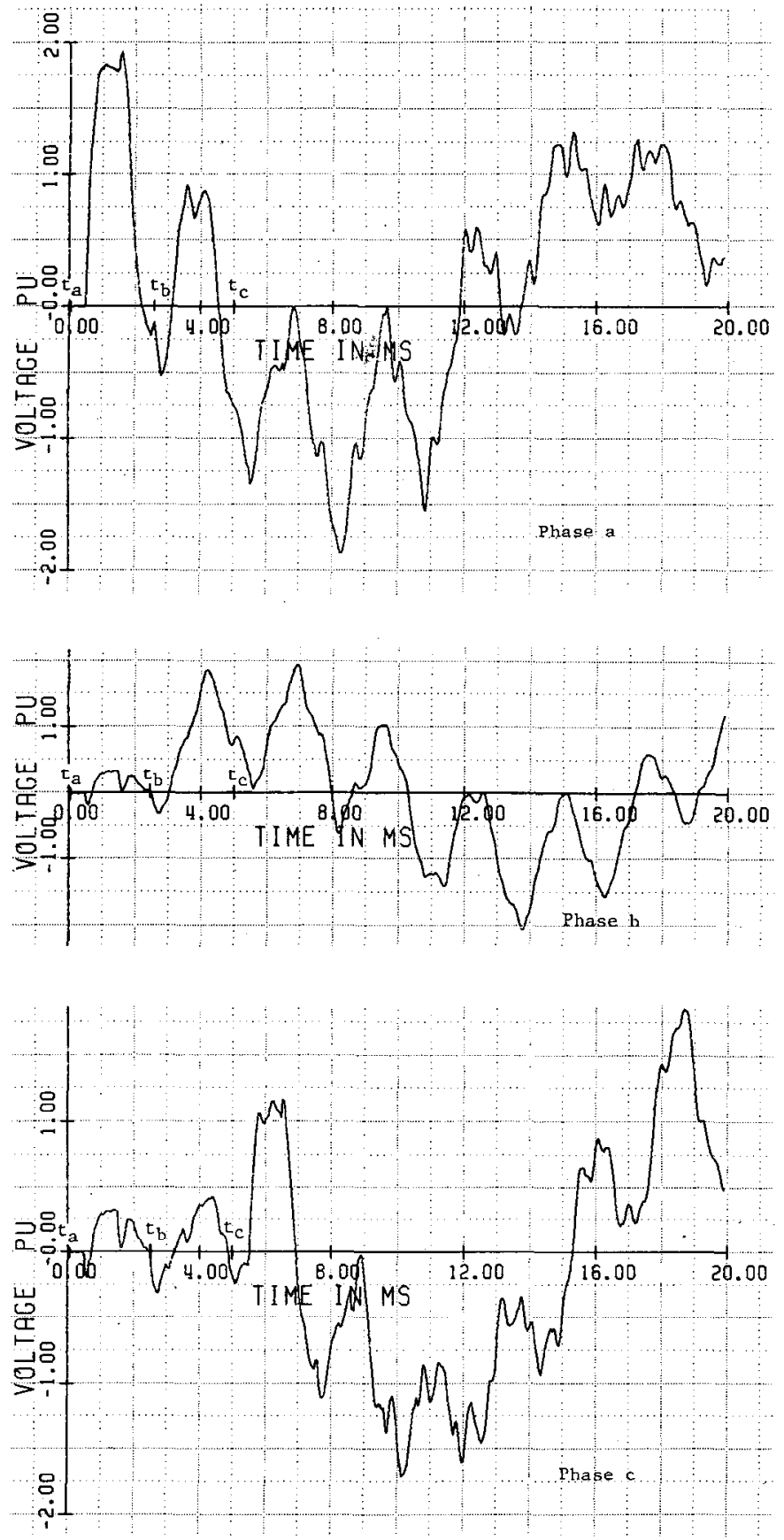

Fig. 10. Receiving end voltages on phases $a, b, c$ at sequential energization of the same line as in Fig. 9 , at $t_{a}=0, t_{b}=2.5 \mathrm{~ms}, t_{c}=5 \mathrm{~ms}$.

nonlinearly interrelated:

$$
\begin{aligned}
\dot{x} & =A x+B u+B^{\prime} u^{\prime} \\
y & =C x+D u+D^{\prime} u^{\prime} \\
y^{\prime} & =E x+F u+F^{\prime} u^{\prime} \\
u^{\prime} & =f\left(y^{\prime}\right)
\end{aligned}
$$

where equation (35) symbolizes a nonlinear feedback. Clearly, the system is now nonlinear and numerical techniques will be needed for the integration. Our main remark here is that small step sizes will be required until the transients attenuate but later they can be significantly increased to an upper limit determined only by the power frequency $\omega_{0}$.

\section{Conclusions}

A new method has been developed for the calculation of transients in power system networks. It is based on expressions of transmission line parameters in terms of the complex frequency $s=\sigma+j \omega$. This permits the approximation of a transfer function $H(s)$ by a rational form for which the poles and residues can easily be calculated by direct search procedures in the $s$-plane.

The resultant rational transfer functions are converted analytically into state equations. The integration of the state equations can be performed either numerically or in closed form. Computational requirements are low both for computing time and storage. No stability or convergence problems have been experienced.

The advantage of the new method appears to be that it permits the powerful tools of state equations and $s$-domain formulations to be applied to realistic transmission lines with possible application of important system theory procedures, such as model reduction (network cquivalencing for transients), optimization (direct calculation of maximized over-voltages), and control. Long range transients can now be calculated advantageously using closed form solutions. On the other hand, the preparatory work for larger systems appears at this stage to be difficult.

\section{REFERENCES}

[1] H. W. Dommel, "Digital computer solution of electromagnetic transients in single and multiphase networks," IEEE Trans. Power App. Syst., vol. PAS-88, Apr. 1969, pp. 388-399.

[2] J. P. Bickford and P. S. Doepel, "Calculation of switching transients with particular reference to line energization," Proc. Inst. Elec. Eng., 114, Apr. 1967, pp. 465-477.

[3] J. R. Carson, "Wave propagation in overhead wires, with ground return," Bell Syst. Tech. J., vol. 5, 1926, pp. 539-554.

[4] W. S. Meyer and H. W. Dommel, "Numerical modelling of frequency-dependent transmission-line parameters in an electromagnetic transients program," IEEE Trans. Power App. Syst., vol. PAS-93, Sept./Oct. 1974, pp. 1401-1409.

[5] A. Semlyen and A. Dabuleanu, "Fast and accurate switching transient calculations on transmission lines with ground return using recursive convolutions," IEEE Trans. Power App. Syst., vol. PAS-94, Mar./Apr. 1975, pp. 561-571.

[6] A. Semlyen, "Contributions to the theory of calculation of electromagnetic transients on transmission lines with frequency dependent parameters," IEEE Trans. Power App. Syst, vol. PAS-100, Feb. 1981, pp. 848-856.

[7] J. R. Marti, "Accurate modelling of frequency-dependent transmission lines in electromagnetic transient simulations," IEEE Trans. Power App. Syst., vol. PAS-101, Jan. 1982, pp. 147-157.

[8] A. Semlyen, "Simulation of electromagnetic transients in power systems using model reduction by rational approximation," in Proc. Canadian Commun. Power Conf., (Montreal, Canada), Oct. 1980, pp. 284-287.

[9] J. R. Wait and K. P. Spies, "On the image representation of the quasi-static fields of a line current source above the ground," Canadian J. Phys., vol. 47, 1969, pp. 2731-2733.

[10] C. Gary, "Approche complète de la propagation multifilaire en haute frequence par utilisation des matrices complexes," EDF Bull. Direction Etudes et Recherches.-Sér. B, no. 3/4, 1976, pp. 5-20.

[11] A. Deri, G. Tevan, A. Semlyen, and A. Castanheira, "The complex ground return plane, a simplified model for homogeneous and multilayer earth return," IEEE Trans. Power App. Syst., vol. PAS100, Aug. 1981, pp. 3686-3693.

[12] A. Semlyen, "Approximation to Carson's loss formulae," Canadian Elec. Eng. J., vol. 6, no. 2, 1981, pp. 30-31. 
[13] L. M. Wedepohl, "Application of matrix methods to the solution of travelling-wave phenomena in polyphase systems," Proc. Inst. Elec. Eng., vol. 110, Dec. 1963 , pp. 2200-2212.

[14] I. S. Gradshteyn and I. M. Ryzhik, Table of Integrals, Series, and Products. New York: Academic Press, 1980.

[15] A. Ralston and P. Rabinowitz, A First Course in Numerical Analysis, 2nd ed. New York: McGraw Hill, 1978.

[16] A. S. Householder, The Numerical Treatment of a Single Non-Linear Equation. New York: McGraw Hill, 1970.

[17] T. Kailath, Linear Systems. Englewood Cliffs, NJ: Prentice Hall, 1980.

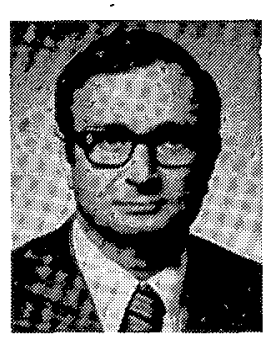

Adam Semlyen (SM'70) received a Dipl. Eng. honours degree in electrical engineering from the Polytechnic Institute of Timisoara, Rumania, and the Dr. Eng. degree from the Polytechnic Institute of Iasi, Rumania.

He started his carrer with the Central Power Station of Timisoara where he was primarily engaged in testing of power equipment, meters, and relays. Simultaneously, he was on the staff of the Faculty of Electrical Engineering of the Polytechnic Institute of Timisoara, teaching a wide range of courses in power engineering. His research interest was in current transformers, power system analysis, and overvoltages in high voltage systems. As a consultant to the Regional Power Authority he designed equipment for compensation of capacitive ground currents. In 1969 he was with Benedikt and Jager, in Vienna, Austria, where he did development studies in reactors and special transformers. He then joined the University of Toronto, Toronto, Ontario, where he is professor in the Department of Electrical Engineering. His current interest is in power system dynamics and electromagnetic transients in transmission lines and systems. He has written a textbook and papers in power systems and related topics.

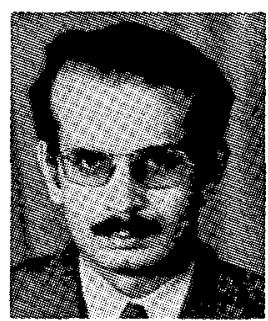

Mansour H. Abdel-Rahman (M'79) was born in Belkas, Egypt on August 9, 1947. He received B.Sc. and M.Sc. degrees from Cairo University in Electrical Engincering in 1970 and 1975, respectively. He received the Ph.D. degree from the University of Manchester, Institute of Science and Technology, United Kingdom in 1979. His Ph.D. topic was in Electric Power Systems. He worked from 1970 to 1976 as an Assistant Lecturer and from 1979 to 1980 as a Lecturer at El-Mansoura University, Egypt and he also worked part-time with the Egyptian Electricity Authority. From 1980 to 1981 he worked as a Research Fellow at the University of Toronto, Canada. He is currently a Visiting Assistant Professor at the University of Windsor, Canada. His interests are in the calculation and assessment of transient phenomena in power systems.

\title{
A Numerical Design Procedure for General Canonic $L C$ One-Ports
}

\author{
STEPHEN E. SUSSMAN-FORT, MEMBER, IEEE
}

\begin{abstract}
A numerical method has been developed and a computer program has been written to design arbitrary, canonic, inductor-capacitor one-ports. The design procedure employs a matrix tridiagonalization method in conjunction with a Newton-Raphson technique to solve for the element values in a canonic $L C$ network to yield a prescribed driving-point impedance function. The numerical method is extremely stable and numerically
\end{abstract}

Manuscript received November 24, 1981, revised April 7, 1982.

The author is with the Department of Electrical Engineering, State University of New York at Stony Brook, Stony Brook, NY 11794. well-conditioned; in one of our examples, a 14th degree, nonplanar $L C$ structure was designed (using single precision arithmetic) yielding element values accurate to six significant figures.

\section{INTRODUCTION}

$\mathrm{N}$ ECESSARY AND SUFFICIENT topological conditions for a lossless one-port to be canonic have recently been discovered [1]. This result is in the form of an existence theorem, and hence does not provide a means for actually calculating the circuit-element values in an arbi- 